\title{
Shot noise in magnetic tunnel junctions from first principles
}

\author{
Kai Liu and Ke Xia \\ Department of Physics, Beijing Normal University, Beijing 100875, China \\ Gerrit E. W. Bauer \\ Institute for Materials Research, Tohoku University, Sendai 980-8577, Japan and Kavli Institute of NanoScience, Delft University \\ of Technology, 2628 CJ Delft, The Netherlands
}

(Received 2 November 2011; revised manuscript received 12 May 2012; published 26 July 2012)

\begin{abstract}
We compute the shot noise in ballistic and disordered $\mathrm{Fe}|\mathrm{MgO}| \mathrm{Fe}$ tunnel junctions by a wave-function-matching method. For tunnel barriers with $\lesssim 5$ atomic layers we find a suppression of the Fano factor as a function of the magnetic configuration. In the antiparallel configuration the shot noise is full up to a threshold bias that indicates the onset of resonant tunneling. We find excellent agreement with recent experiments when interface disorder is taken into account.
\end{abstract}

DOI: 10.1103/PhysRevB.86.020408

PACS number(s): 72.25.Ba, 72.10.Bg, 85.75.-d

The statistics of electron transport in mesoscopic systems has been the subject of intensive research in the past decades, leading to important and useful insights. ${ }^{1,2}$ In a two-terminal conductor with a time-dependent current $I(t)$, the simplest measure is the noise power $P(\omega)=\int_{-\infty}^{\infty}\langle\triangle I(0) \triangle I(t)\rangle e^{i \omega t} d t$, where $\triangle I(t) \equiv I(t)-\langle I\rangle$ denotes the instantaneous fluctuation from the average current and $\langle\cdots\rangle$ is a time and statistical average. The shot noise $S$ is the zero frequency limit of the noise power when the applied voltage $|e V|$ is sufficiently larger than the thermal energy $k_{B} T$. The classical shot noise characterized by an uncorrelated Poissonian process is given by the Schottky formula $S=2 e\langle I\rangle .^{3}$ Shot noise contains information about the charge of the elementary excitations, entanglement, the wave versus particle nature of electron transport, and provides a diagnostic for open transport channels. ${ }^{4}$

Magnetic tunnel junctions (MTJs) with $\mathrm{MgO}$ barriers 5,6 have great potential for applications in magnetic random access memory elements and high-frequency generators. ${ }^{7-10}$ Band structure calculations of isomorphic $\mathrm{Fe}|\mathrm{MgO}| \mathrm{Fe}$ layered structures predicted a large drop in the electric resistance when the relative magnetization direction of the two ferromagnets switches from antiparallel to parallel. ${ }^{11,12}$ The subsequently observed large Tunnel Magnetoresistance Ratio (TMR) ${ }^{5,6}$ can be explained in terms of the symmetry matching of only the majority-spin states in $\mathrm{Fe}$ with the $\triangle_{1}$ band of $\mathrm{MgO}$, which is by far the least evanescent in the gap. The tunneling ratio of the majority-spin electrons is therefore relatively high while minority-spin states are efficiently filtered out by the $\mathrm{MgO}$ barrier. However, a quantitative first-principles description of transport in magnetic tunneling junctions is complicated by defects. The chemical composition of the interface strongly affects the TMR, ${ }^{13-15}$ and various interfacial defects have been identified to reduce the TMR. ${ }^{16-18}$ The $I-V$ curves alone cannot discriminate between the possible different origins that reduce the TMR.

According to conventional wisdom, shot noise in tunnel junctions is classical, ${ }^{1}$ in agreement with earlier experiments. ${ }^{19-21}$ Recent evidence that shot noise in MTJs is suppressed in the parallel configuration was very surprising. ${ }^{22}$ In order to resolve this issue we present parameter-free calculations of shot noise in magnetic tunnel junctions. We compute sub-Poissonian shot noise for the parallel magnetic configuration and explain the results in terms of highly transmitting resonant tunneling between states localized at the interfaces between the ferromagnet and insulator. The agreement between first-principles theory and experiments ${ }^{22}$ is quantitative when disorder is taken into account. These results provide strong evidence of coherent transport and (additional) proof for the very high quality of the MTJs used in that study.

According to the scattering theory of transport, a twoterminal conductor subjected to a sufficiently small bias voltage $V$ leads to a time-averaged electric current

$$
\langle I\rangle=\frac{e^{2}}{h}|e V| \operatorname{Tr}\left(\mathbf{t}^{\dagger} \mathbf{t}\right)
$$

and shot noise

$$
S=\frac{2 e^{2}}{h}|e V| \operatorname{Tr}\left(\mathbf{r}^{\dagger} \mathbf{r t}^{\dagger} \mathbf{t}\right),
$$

where $\mathbf{t}$ and $\mathbf{r}$ are the matrices of the transmission and reflection coefficients in the space of the transport channels of the leads to the scattering region. These equations become more transparent by making use of the distribution function $\rho(T)=$ $\sum_{n} \delta\left(T-T_{n}\right)$ of the eigenvalues $\left\{T_{n}\right\}$ of the transmission matrix $\mathbf{T}=\mathbf{t}^{\dagger} \mathbf{t}$, where $T_{n} \in[0,1]$ :

$$
\begin{gathered}
\langle I\rangle=\frac{e^{2}}{h}|e V| \int \rho(T) T d T, \\
S=\frac{2 e^{2}}{h}|e V| \int \rho(T) T(1-T) d T=2 e F\langle I\rangle,
\end{gathered}
$$

where $F \leqslant 1$ is the Fano factor. For a conventional tunnel junction the transmissions are small and $\rho(T)$ is substantial only for $T \ll 1$. We then may disregard the $\sim T^{2}$ term in the integrand of Eq. (4) and classical shot noise corresponding to $F \rightarrow 1$ is recovered. Clearly, a suppression of the shot noise that would correspond to a Fano factor that is significantly smaller than unity requires that $\rho(T)$ is significant at transmissions close to unity. Indeed, below we find such highly transmitting states in MTJs with sufficiently thin barriers.

Previous theoretical treatments of the statistics of quantum transport have been limited to simple models. While these can be sufficiently accurate for, e.g., structures defined on a twodimensional electron gas, the details of the electronic structure 


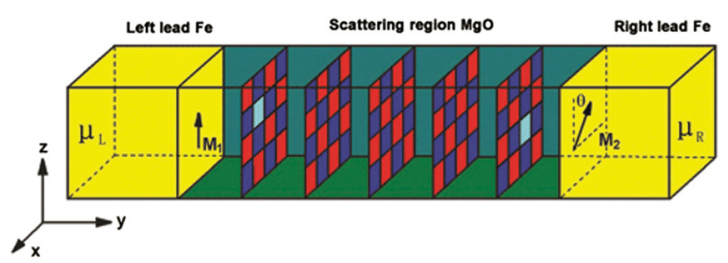

FIG. 1. (Color online) Schematic diagram of a $\mathrm{Fe}|\mathrm{MgO}| \mathrm{Fe}(001)$ junction containing five $\mathrm{MgO}$ monolayers. The magnetization $\mathbf{M}_{1}$ of the left lead coincides with the $z$ axis; the magnetization $\mathbf{M}_{2}$ of the right lead lies in the $x z$ plane with angle $\theta$. The red and blue grids in the scattering region denote the $\mathrm{O}$ and $\mathrm{Mg}$ atoms, respectively. We allow for interfacial disorder (oxygen vacancies, cyan grid) at the $\mathrm{Fe} \mid \mathrm{MgO}$ interfaces. The applied bias $e V=\mu_{R}-\mu_{L}$, where $\mu_{R}$ and $\mu_{L}$ are the chemical potentials of the right and left leads, respectively.

are essential to understand (nearly) ballistic MTJs. ${ }^{11,12,23}$ This Rapid Communication reports the results of material-specific first-principles calculations of the statistics of transport in $\mathrm{Fe}|\mathrm{MgO}| \mathrm{Fe}$ magnetic tunnel junctions as a function of magnetic configuration, voltage bias, and interface morphology, and compares theory with experiments by Arakawa et al.,22 in particular, the suppression of the Fano factor for the parallel configuration.

We consider an MTJ consisting of a $\mathrm{MgO}$ barrier and two semi-infinite iron leads as shown in Fig. 1. The electric current flows along the (001) crystal growth direction. We incorporate the small lattice mismatch between the leads and the barrier by a $3 \%$ compression of the $\mathrm{MgO}$ lattice constant. The self-consistent calculations are carried out with the tight-binding linear muffin-tin orbital (TB-LMTO) ${ }^{24}$ surface Green's function method. ${ }^{25}$ Since a fully self-consistent calculation for each random configuration is prohibitively expensive, the potentials on each atom are computed by the layer coherent potential approximation (CPA) ${ }^{26}$ The CPA atomic sphere (AS) potentials serve as the input to the second step, in which the transmission matrix is calculated using a TB-LMTO implementation. ${ }^{27}$ This procedure was found to be very accurate when comparing CPA potentials and the corresponding transport properties with those from fully self-consistent supercell calculations for small lateral supercells. Disorder is then modeled by large lateral supercells, distributing the self-consistently calculated CPA-AS potentials randomly layer for layer in the appropriate concentrations in as many configurations as necessary. Depending on the defect concentration, most of our results are based on lateral supercells containing 72 (two times $6 \times 6$ ) or 128 (two times $8 \times 8) \mathrm{Fe}$ atoms per monolayer. In our calculations for clean junctions, we use in the whole two-dimensional
(2D) Brillouin zone $6.4 \times 10^{4} k$ points for the junctions with three, four, and five layers of $\mathrm{MgO}$ and as many as $1.44 \times 10^{5} k$ points for the junction with seven layers of $\mathrm{MgO}$. This $k$ mesh is fine enough to reduce the error bar in conductance and Fano factor to less than $1 \%$. For dirty junctions, we use $400 k$ points in the whole $2 \mathrm{D}$ Brillouin zone and 20 configurations for the 5-monolayer (ML) $\mathrm{MgO}$ junction and $100 k$ points and five configurations for other disorder calculations. The convergence for the conductance is estimated around 5\%, while for the Fano factor it is smaller than $1 \%$.

Here, TMR $\equiv R(\mathrm{AP}) / R(\mathrm{P})-1$, where $R(\mathrm{AP})[R(\mathrm{P})]$ is the electric resistance for the antiparallel (AP) [parallel (P)] configuration of the lead magnetizations. For five $\mathrm{MgO}$ monolayers $(L)$ at low bias $\mathrm{TMR}=3580 \%$ for specular interfaces, which decreases drastically to $250 \%$ when $5.56 \%$ oxygen vacancies (OV, the energetically most favorable defect) are introduced at both interfaces. The TMR for the ideal junction is consistent with published calculations, ${ }^{11}$ while that for disordered junctions is of the same order of magnitude as found in experiments. ${ }^{5}$

Based on the calculated scattering matrix, we compute the Fano factor for various junction parameters. The results are shown in Table I, together with the TMR. For thick barriers, the Fano factors are very close to unity, implying full Poisson noise as expected. As the barrier gets thinner, the Fano factor of the parallel configuration is increasingly suppressed. For a five-MgO-layer junction with $5.56 \%$ interfacial disorder, the Fano factor is $F_{\mathrm{P}}=0.87(4)$ and $F_{\mathrm{AP}}=0.98(1)$ for both configurations, close to the experimental values $F_{\mathrm{P}}=0.91(2)$ and $F_{\mathrm{AP}}=0.98(1)$ for the same thickness. ${ }^{22}$ We can identify the majority- $(\uparrow)$ and minority- $(\downarrow)$ spin contributions to be $F_{\mathrm{P}}^{\uparrow}=0.96$ and $F_{\mathrm{P}}^{\downarrow}=0.72$, where $F_{\mathrm{P}}^{\uparrow(\downarrow)} \equiv S_{\mathrm{P}}^{\uparrow(\downarrow)} /\left(2 e\left\langle I_{\mathrm{P}}^{\uparrow(\downarrow)}\right\rangle\right)$.

In order to trace the origin of the shot noise suppression, we plot the distribution functions of the transmission matrix eigenvalues $\rho_{\mathrm{P} / \mathrm{AP}}(T)$ in Fig. 2 involving $7 \times 10^{6}$ eigenvalues over the whole Brillouin zone. For P, we identify a few high values of $T_{n}$, which, according to Eqs. (3) and (4), affect shot noise $S$ more strongly than conductance $G: 0.3 \%$ of the eigenvalues are larger than 0.05 but contribute about $39 \%$ to $G$ but $89 \%$ to the integrand proportional to $T^{2}$ in Eq. (4), which suppresses $S$. The integrands proportional to $T$ and $T^{2}$ are shown for each eigenvalue interval in the histograms of Fig. 2. The dashed bars indicate a larger statistical error caused by the small number of eigenvalues at high $T_{n}$. For AP, only very few $T_{n}$ fall into the region between 0.05 and 0.1 , and the rest $(99.95 \%)$ are all less than 0.05 .

In Table I we can see that interfacial defects are necessary to explain the observed shot noise suppression. ${ }^{22}$ The OV

TABLE I. Barrier thickness dependence of the Fano factor in Fe $|n \mathrm{MgO}| \mathrm{Fe}$ MTJs for the parallel (P) and antiparallel (AP) configuration. The results in square brackets are obtained for disordered junctions with 5.56\% oxygen vacancies at the interfaces, where the error bar is given in parentheses.

\begin{tabular}{lcccc}
\hline \hline Fano factor & $3 \mathrm{MgO}$ & $4 \mathrm{MgO}$ & $5 \mathrm{MgO}$ & $7 \mathrm{MgO}$ \\
\hline $\mathrm{P}$ & $0.64[0.65(2)]$ & $0.91[0.69(4)]$ & $0.97[0.87(4)]$ & $1.00[0.99(1)]$ \\
$\mathrm{AP}$ & $0.94[0.77(2)]$ & $1.00[0.94(1)]$ & $1.00[0.98(1)]$ & $1.00[0.99(1)]$ \\
TMR & $1320 \%[165 \%]$ & $2400 \%[288 \%]$ & $3580 \%[250 \%]$ & $5600 \%[107 \%]$ \\
\hline \hline
\end{tabular}




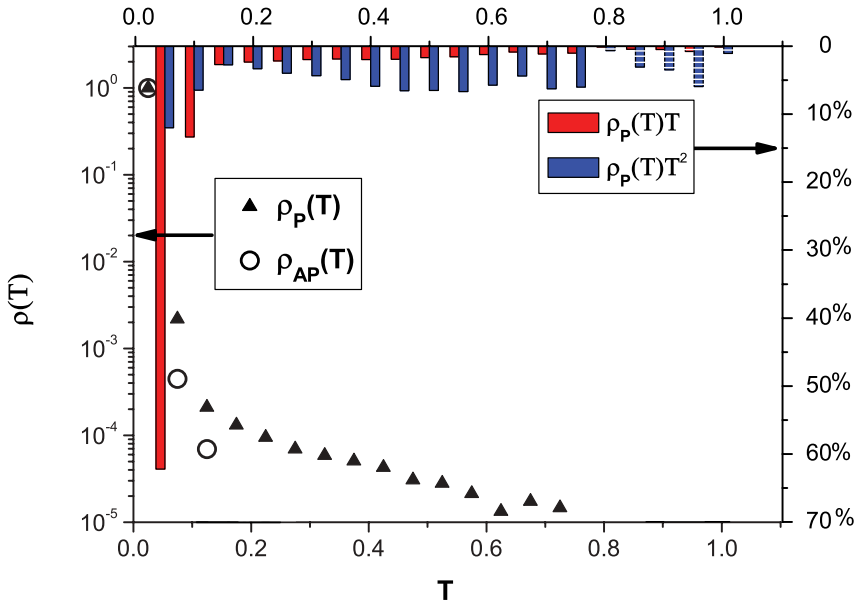

FIG. 2. (Color online) Distribution of the transmission matrix eigenvalues $\rho(T)$ at Fermi level. The open circles are obtained for the AP configuration, for which $99.95 \%$ of eigenvalues $T_{n}<0.05$. Solid triangles represent results for the $\mathrm{P}$ configuration. While most eigenvalues are still less than 0.05 , the few high values prove the presence of resonant tunneling states. The red and blue histograms indicate the contribution of the integrands $T$ and $T^{2}$ to each eigenvalue interval (for P). Dashed bars indicated increased statistical error due to the small number of $T_{n}$ 's approaching unity.

concentration-dependent Fano factor for the five-MgO-layer junction can be found in Table II. The statistics of our supercell calculation is found to be good in comparison with a Green's function formalism in which impurity scattering is handled by the CPA. ${ }^{28}$ Furthermore, even though an OV concentration of around 5\% suppresses the Fano factor, a further increase leads to a remarkable recovery of the full shot noise. The full shot noise observed in earlier experiments ${ }^{20,21}$ is therefore consistent with higher disorder. Notice that when the OVs are in the middle of barrier, conductance decreases. ${ }^{17,29}$ But an $\mathrm{OV}$ in $\mathrm{MgO}$ is likely to stay at the $\mathrm{Fe} \mid \mathrm{MgO}$ interface due to compressive strain during crystal growth. ${ }^{15}$ Here we find a conductance that increases when OVs are introduced at the interfaces of $\mathrm{Fe} \mid \mathrm{MgO}$, effectively reducing the width of the $\mathrm{MgO}$ tunnel barrier. ${ }^{18}$

In order to understand the sensitivity to the OVs, we plot the energy dependence of the conductance of five $\mathrm{MgO}$ MTJs with different OVs concentrations in Fig. 3. In ballistic junctions the minority-spin conductance for $\mathrm{P}$ and the AP conductance are strongly suppressed. Below the Fermi energy

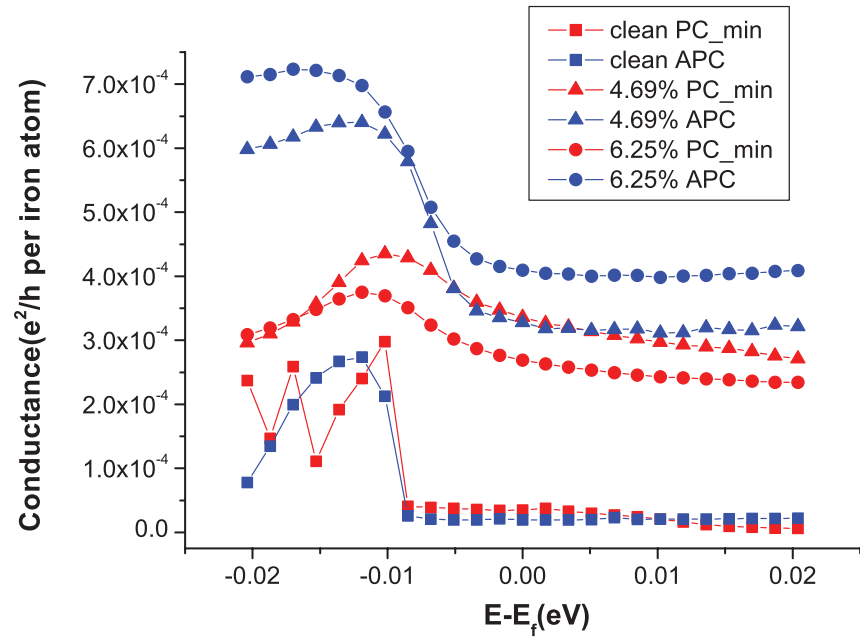

FIG. 3. (Color online) Energy-dependent conductance for $\mathrm{P}$ (minority spin) and AP configuration of a $5 \mathrm{MgO}$ MTJ for different impurity concentrations. Solid squares represent the ballistic junction, triangles $4.69 \%$ OV disorder, and circles $6.25 \%$ OV disorder. Red symbols stand for the minority-spin conductance in the $\mathrm{P}$ and blue ones for the conductance in the AP configuration.

high transmissions are observed, however. For $\mathrm{P}$ these are caused by the overlap between interface states on both sides of the barrier. For AP, the interface states exist only on one side of the barrier, but since their symmetry is not orthogonal to the $\Delta_{1}$ states in the barrier and the majority-spin states on the another side, the conductance is still high. Small amounts of OVs broaden and shift highly transmitting resonant channels toward the Fermi energy, thereby suppressing $F_{\mathrm{P}}$ (and the TMR). However, a further increase of the disorder destroys the resonant channels, thereby recovering the full shot noise. The AP peak disappears and becomes a step structure near the Fermi energy.

A $5 \%$ OV concentration appears to be close to the experiment, ${ }^{22}$ since it explains both Fano factors and the TMR. Further information may be gained by the $\theta$ dependence of the Fano factor in Fig. 4 for five $\mathrm{MgO}$ junctions with $5.56 \%$ OVs. $F(\theta)$ increases when moving from $\mathrm{P}$ to $\mathrm{AP}$, which can be understood by the arguments above.

An important issue of the current-induced spin transfer torque (STT) in MTJs is its bias dependence. Recent experiments $^{30}$ discovered a nonlinear increase of the STT

TABLE II. OV concentration dependence of the Fano factor in five MgO MTJs for P and AP configurations. The impurity concentrations are obtained by different numbers of OVs at the interfaces per lateral unit cell. Two OVs in the $6 \times 6$ supercell correspond to $5.56 \%$, and three or four OVs in an $8 \times 8$ supercell correspond to $4.69 \%$ and $6.25 \%$, respectively. The conductances are given in units of $10^{-5} e^{2} / h$ per Fe atomic interfacial area, where the statistical error bar is given in parentheses. The conductances in square brackets are obtained by the Green's function method (Ref. 28) as a check of the statistics of the supercell method.

\begin{tabular}{|c|c|c|c|c|c|}
\hline \multirow[b]{2}{*}{ Concentration } & \multicolumn{3}{|c|}{$\mathrm{P}$} & \multicolumn{2}{|c|}{ AP } \\
\hline & $G(\mathrm{maj})$ & $G(\min )$ & Fano factor & $G$ & Fano factor \\
\hline 0 & $68.00[68.50]$ & $3.47[3.51]$ & 0.97 & $1.95[1.95]$ & 1.00 \\
\hline $4.69 \%$ & 89(3)[94.5] & $41(7)[33.6]$ & $0.85(3)$ & $35(3)[32.8]$ & $0.98(1)$ \\
\hline $5.56 \%$ & $80(4)[91.0]$ & $44(10)[29.2]$ & $0.87(4)$ & $36(4)[37.5]$ & $0.98(1)$ \\
\hline $6.25 \%$ & $79(3)[85.5]$ & $25(4)[26.9]$ & $0.95(2)$ & $38(3)[41.0]$ & $0.98(1)$ \\
\hline
\end{tabular}




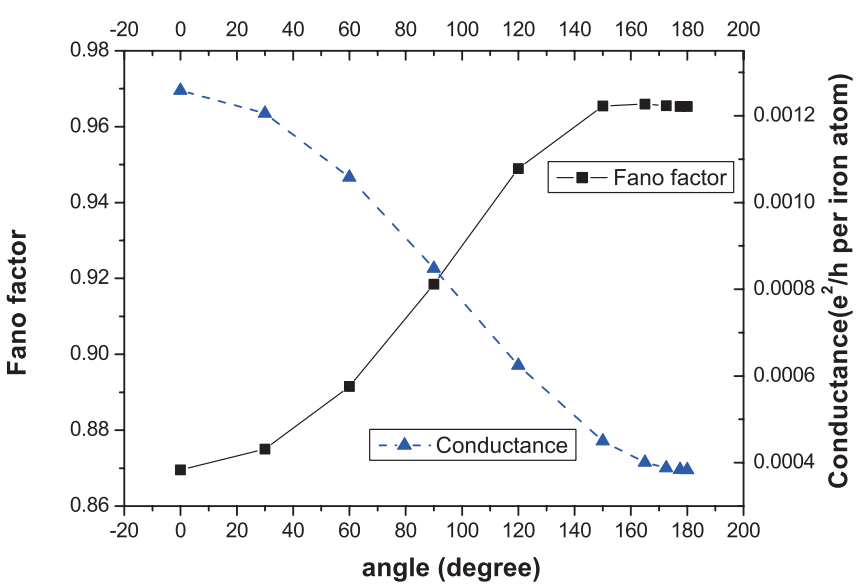

FIG. 4. (Color online) Angular-dependent Fano factor (squares) and conductance (triangles, per Fe atom at the interface) of five $\mathrm{MgO}$ MTJs with $5.56 \%$ OV disorder at the interfaces. When $\theta$ changes from $\mathrm{P}$ to AP, the conductance decreases monotonously and the Fano factor increases.

and current at an applied bias of $0.2 \mathrm{~V}$. This value is far below the $\mathrm{MgO}$ band gap, as calculated in the local density approximation (LDA). ${ }^{23}$ Since the LDA strongly underestimates band gaps, the observed threshold must have a different origin. A recent first-principles analysis of the STT in $\mathrm{Fe}|\mathrm{MgO}| \mathrm{Fe}$ junctions explained the threshold in terms of resonant transmission channels in the AP configuration. ${ }^{31}$ This hypotheses can be tested by the bias dependence of the shot noise. At finite bias $V$, the zero temperature current and shot noise read ${ }^{1}$

$$
\begin{array}{r}
I(V)=\frac{e^{2}}{h} \int^{e V}\left[\int \rho(V, T, E) T d T\right] d E, \\
S(V)=\frac{2 e}{h} \int^{e V}\left[\int \rho(V, T, E) T(1-T) d T\right] d E .
\end{array}
$$

Figure 5 shows the integrated current and Fano factor as a function of applied bias for an $\mathrm{Fe}(\uparrow)|5 \mathrm{MgO}| \mathrm{Fe}(\downarrow)$ junction. The Fano factor is unity for low bias but suddenly decreases with increasing bias at the threshold of the nonlinear current characteristic, which for a clean junction is at about $0.8 \mathrm{~V}$, consistent with the computed threshold bias in the STT. Small amounts of oxygen vacancies in $\mathrm{MgO}$ can lower this threshold bias to values closer to the experiment. ${ }^{31}$

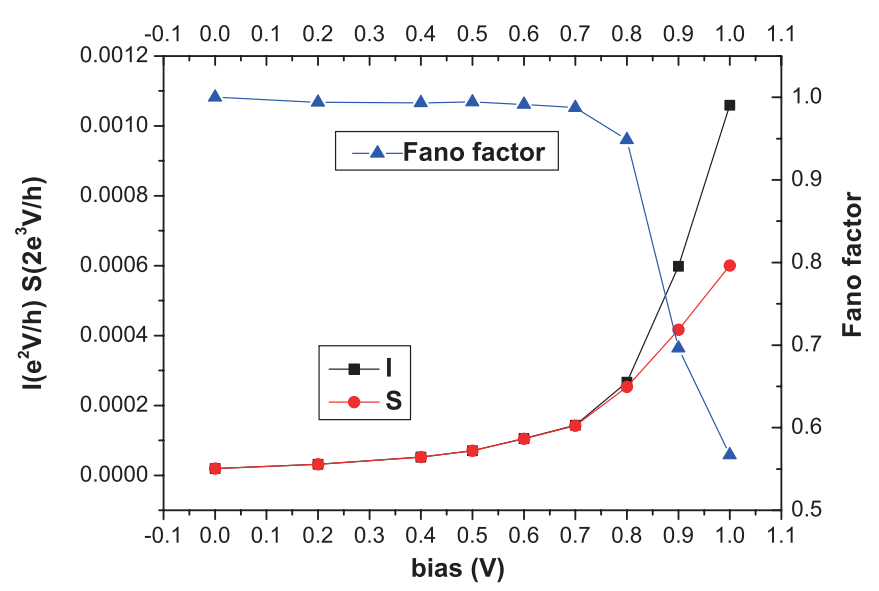

FIG. 5. (Color online) Bias voltage-dependent Fano factor of an antiparallel five-MgO-layer MTJ. $I$ and $S$ stand for current (per Fe atom at the interface) and shot noise integrated over the bias window, respectively.

In conclusion, we compute sub-Poissonian shot noise for magnetic junctions with thin $\mathrm{MgO}$ barriers from first principles, which is in good agreement with experiments. We interpret these results as strong evidence for resonant tunneling states that are weakly broadened by disorder scattering. While it was known that $\mathrm{MgO}$ based tunneling junctions can be grown with high crystalline quality, we believe that the implied wave function coherence over the tunneling barrier is an important piece of information that lends credibility to the prediction of large thermal spin transfer torques. ${ }^{32}$ This additional evidence of the superior electronic properties of $\mathrm{MgO}$ tunneling barriers should have ramifications for the application of this materials to other than ferromagnetic systems, such as Josephson junctions.

Note added in proof. Recently we became aware of Tanaka et al. ${ }^{33}$ who reported shot noise measurements to detect coherent tunneling in spinel-based MTJs.

We gratefully acknowledge financial support from $\mathrm{Na}$ tional Basic Research Program of China under Grant No. 2011CB921803, 2012CB921304, NSF-China, the Dutch FOM Foundation, DFG Priority Program "SpinCat", and EU-ICT-7 Contract Mo. 257159 MACALO. We thank the authors of Ref. 22 for valuable discussions concerning their experimental results.
${ }^{1}$ Ya. M. Blanter and M. Büttiker, Phys. Rep. 336, 1 (2000).

${ }^{2}$ Quantum Noise in Mesoscopic Physics, edited by Yu. V. Nazarov (Kluwer, Dordrecht, 2003).

${ }^{3}$ W. Schottky, Ann. Phys. (Leipzig) 57, 541 (1918).

${ }^{4}$ C. Beenakker and C. Schönenberger, Phys. Today 56(5), 37 (2003).

${ }^{5}$ S. Yuasa, T. Nagahama, A. Fukushima, Y. Suzuki, and K. Ando, Nat. Mater. 3, 868 (2004).

${ }^{6}$ S. S. P. Parkin, C. Kaiser, A. Panchula, P. M. Rice, B. Hughes, M. Samant, and S.-H. Yang, Nat. Mater. 3, 862 (2004).
${ }^{7}$ S. C. Oh, S. Y. Park, A. Manchon, M. Chshiev, J. H. Han, H.-W. Lee, J.-E. Lee, K.-T. Nam, Y. Jo, Y.-C. Kong, B. Dieny, and K.-J. Lee, Nat. Phys. 5, 898 (2009).

${ }^{8}$ A. M. Deac, A. Fukushima, H. Kubota, H. Maehara, Y. Suzuki, S. Yuasa, Y. Nagamine, K. Tsunekawa, D. D. Djayaprawira, and N. Watanabe, Nat. Phys. 4, 803 (2008).

${ }^{9}$ M. H. Jung, S. Park, C.-Y. You, and S. Yuasa, Phys. Rev. B 81, 134419 (2010)

${ }^{10}$ R. Matsumoto, A. Fukushima, K. Yakushiji, S. Yakata, T. Nagahama, H. Kubota, T. Katayama, Y. Suzuki, K. Ando, S. Yuasa, B. Georges, V. Cros, J. Grollier, and A. Fert, Phys. Rev. B 80, 174405 (2009). 
${ }^{11}$ W. H. Butler, X.-G. Zhang, T. C. Schulthess, and J. M. MacLaren, Phys. Rev. B 63, 054416 (2001).

${ }^{12}$ J. Mathon and A. Umerski, Phys. Rev. B 63, 220403 (2001).

${ }^{13}$ J. M. De Teresa, A. Barthélémy, A. Fert, J. P. Contour, F. Montaigne, and P. Seneor, Science 286, 507 (1999).

${ }^{14}$ G. X. Miao, Y. J. Park, J. S. Moodera, M. Seibt, G. Eilers, and M. Münzenberg, Phys. Rev. Lett. 100, 246803 (2008).

${ }^{15}$ P. G. Mather, J. C. Read, and R. A. Buhrman, Phys. Rev. B 73, 205412 (2006).

${ }^{16}$ P. X. Xu, V. M. Karpan, K. Xia, M. Zwierzycki, I. Marushchenko, and P. J. Kelly, Phys. Rev. B 73, 180402(R) (2006).

${ }^{17}$ J. P. Velev, K. D. Belashchenko, S. S. Jaswal, and E. Y. Tsymbal, Appl. Phys. Lett. 90, 072502 (2007).

${ }^{18}$ Y. Ke, K. Xia, and H. Guo, Phys. Rev. Lett. 105, 236801 (2010).

${ }^{19}$ R. Guerrero, F. G. Aliev, Y. Tserkovnyak, T. S. Santos, and J. S. Moodera, Phys. Rev. Lett. 97, 266602 (2006).

${ }^{20}$ R. Guerrero, D. Herranz, F. G. Aliev, F. Greullet, C. Tiusan, M. Hehn, and F. Montaigne, Appl. Phys. Lett. 91, 132504 (2007).

${ }^{21}$ K. Sekiguchi, T. Arakawa, Y. Yamauchi, K. Chida, M. Yamada, H. Takahashi, D. Chiba, K. Kobayashi, and T. Ono, Appl. Phys. Lett. 96, 252504 (2010).
${ }^{22}$ T. Arakawa, K. Sekiguchi, S. Nakamura, K. Chida, Y. Nishihara, D. Chiba, K. Kobayashi, A. Fukushima, S. Yuasa, and T. Ono, Appl. Phys. Lett. 98, 202103 (2011).

${ }^{23}$ C. Heiliger and M. D. Stiles, Phys. Rev. Lett. 100, 186805 (2008).

${ }^{24}$ O. K. Andersen, Z. Pawlowska, and O. Jepsen, Phys. Rev. B 34, 5253 (1986).

${ }^{25}$ I. Turek, V. Drchal, J. Kudrnovsky, M. Sob, and P. Weinberger, Electronic Structure of Disordered Alloys, Surfaces and Interfaces (Kluwer, Boston, 1997).

${ }^{26}$ P. Soven, Phys. Rev. 156, 809 (1967).

${ }^{27}$ K. Xia, M. Zwierzycki, M. Talanana, P. J. Kelly, and G. E. W. Bauer, Phys. Rev. B 73, 064420 (2006).

${ }^{28}$ Y. Ke, K. Xia, and H. Guo, Phys. Rev. Lett. 100, 166805 (2008).

${ }^{29}$ X.-G. Zhang, Yan Wang, and X. F. Han, Phys. Rev. B 77, 144431 (2008).

${ }^{30}$ C. Wang, Y.-T. Cui, J. A. Katine, R. A. Buhrman, and D. C. Ralph, Nat. Phys. 7, 496 (2011).

${ }^{31}$ X. Jia, K. Xia, Y. Ke, and H. Guo, Phys. Rev. B 84, 014401 (2011).

${ }^{32}$ X. Jia, Ke Xia, and G. E. W. Bauer, Phys. Rev. Lett. 107, 176603 (2011).

${ }^{33}$ T. Tanaka et al., Appl. Phys. Express 5, 053003 (2012). 